\title{
INTRAUTERINE INFECTION AND THE EYE
}

\author{
ISABELLE RUSSELL-EGGITT $^{1}$ and SUSAN LIGHTMAN ${ }^{2}$ \\ London
}

\begin{abstract}
SUMMARY
This paper reviews the manifestations of intrauterine infection with toxoplasma gondii, rubella, cytomegalovirus, herpes simplex, varicella-zoster and syphilis with particular emphasis on the ocular findings.
\end{abstract}

Intrauterine infection is a major cause of inflammation in the neonatal eye. Infection in utero can result in resorption of the embryo, abortion or stillbirth. If the infant survives it may be born prematurely, suffer from intrauterine growth retardation, be malformed, scarred and the infection may be still active. Microorganisms may be teratogenic by causing cell death, alteration in cell growth or chromosomal damage. The varicella-zoster virus, CMV and rubella probably act in a combination of these ways. Inflammation with subsequent tissue destruction are probably the major causes of structural abnormalities in congenital syphilis and toxoplasma infection. The infection may persist after birth continuing to cause damage in development and risking infection of other infants.

Certain infective agents such as toxoplasma, rubella, cytomegalovirus and herpes simplex often produce a similar clinical picture of congenital infection. ${ }^{1}$ Common features are purpura, jaundice, anaemia, microcephaly/ hydrocephaly, cerebral calcification, pneumonitis, microphthalmia, cataract and chorioretinitis. Whilst any of these abnormalities may occur some are commoner in one condition than another; for instance, cataracts are common in rubella, but are rare in toxoplasmosis and then often in association with retinal detachment. The ophthalmologist plays a role in diagnosis of the systemic illness as well as management of ocular problems.

It is usually a primary infection of the mother that harms the fetus. However recurrent disease in spite of some maternal immunity may be a cause of congenital infection.

\section{HERPES SIMPLEX}

The DNA of types 1 and 2 of herpes simplex are very

'Hospitals for Sick Children, Great Ormond Street, London WC1.

${ }^{2}$ Moorfields Eye Hosptial, City Road, London EC1V 2PD.

Correspondence to: I. M. Russell-Eggitt FRCS, FCOphth, Hospitals for Sick Children, Great Ormond Street, London WC1. similar with considerable overlap in the cross-reactivity. Primary infection with herpes simplex is uncommon in pregnancy and may be asymptomatic or limited to a mild pharyngitis with a cutaneous vesicular eruption. Fetal infection with herpes simplex is often fatal usually resulting in abortion, but if the fetus survives and is born, skin vesicles or scarring, microcephaly or hydrancephaly, encephalitis, chorioretinitis and hepatitis can occur. Severe disease can result from fetal infection at any time during gestation.

Like other herpes viruses immunosuppression lights up latent infection. Most maternal herpes simplex infection is a localised recurrent genital vesicular eruption. Herpes simplex infection in neonates is therefore mostly acquired by infection at delivery and type 2 is most commonly responsible. The mortality of newborn infants infected with herpes simplex is high and for this reason caesarean sections are now often performed in women with active genital lesions. Many of the mothers howerver, may be asymptomatic at the time of delivery, leading to infection of the newborn infant and a delay in making the diagnosis.

Neonatal herpes simplex mostly manifests as a cutaneous vesicular eruption progressing to generalised infection in $50 \%$ of cases, with hepatitis, pneumonia and disseminated intravascular coagulation. However, not all infants have skin lesions and the ophthalmologist may be the first to suggest the diagnosis if the typical retinitis is seen. If the infection is acquired at birth the retinitis may not be seen until at least one month of age.

In acute ocular involvement there usually are epithelial lesions of the lids, conjunctiva and cornea. In the neonate herpes conjunctivitis is not accompanied by follicles and there is often only mild injection. Stromal keratitis occurs without antiviral therapy of the dendritic lesions. Cataracts may also occur and virus could be cultured from the lens aspirate in an 18 month old child who also had oval pigmented chorioretinal scars at both temporal equators. ${ }^{2}$ Retinitis with vitritis rarely occurs, but then is often severe, and uveitis may result in secondary cataract formation. Treatment is with intravenous acyclovir and topical therapy for keratitis.

The late neurological sequelae of neonatal herpes simplex infection includes: spasticity, microcephaly, psy- 
chomotor retardation, leaming difficulties, cortical blindness and sensorineural hearing loss. ${ }^{3}$ El Azazi reviewed the late ocular manifestations of neonatal herpes simplex in 32 children. ${ }^{4}$ Findings included: cortical visual loss, squint, chorioretinal scars, optic atrophy, corneal scarring and cataract. Typically the fundus lesions are less pigmented than in toxoplasmosis as the primary pathology is in the retina, which atrophies with pigmentation of the surrounding and underlying choroid. The majority of those with eye lesions are also neurologically impaired.

\section{CYTOMEGALOVIRUS (CMV)}

The CMV viruses are the largest members of the herpes virus family. The human CMV appears to be species specific, and replicates much slower than herpes simplex. Antibodies to CMV are present in just over $50 \%$ of women of childbearing age in Europe, but in up to $90 \%$ in more densely populated regions of the world. ${ }^{5}$

Most infections with CMV are subclinical including those acquired in utero and perinatally. CMV is probably the commonest cause of intrauterine infection in the developed world, with approximately $1 \%$ of newborn infants excreting live virus at birth. In utero infection with CMV can occur both from primary and from recurrent CMV and therefore can occur in consecutive pregnancies. However, infants born to mothers with a recurrent CMV infection very rarely $(<1 \%)$ have clinically apparent disease. About half the mothers with a primary CMV infection (when maternal IgM antibody to CMV is found) transmit virus to their fetus, but only $10-15 \%$ of these are born with clinically apparent disease. ${ }^{5}$

It is not understood why only some infants have manifest disease and infants with congenital CMV have been used as a model for understanding virus latency. These infants seem to have a specific defect in CMV-induced lymphocyte proliferation. ${ }^{6} \mathrm{CMV}$ is probably the commonest intrauterine infection in the western world, but uncommonly causes morbidity. However infants born to HIV seropositive mothers may succumb to CMV encephalitis. ${ }^{\text {? }}$

Stagno summarised the acute findings in 34 newborns with congenital CMV infection. ${ }^{8}$ Petechiae were apparent in the majority by two weeks of age. The liver was the most common organ involved and they tended to be born small and prematurely. Half were microcephalic, but only four had chorioretinitis. This is similar to Ramsay's findigns in 65 infants who were born with symptomatic CMV and were reviewed at more than three years of age. ${ }^{9}$ Only $29(45 \%)$ had neurological impairment and just under a quarter of these had isolated sensorineural deafness. Only seven $(11 \%)$ were known to have ocular morbidity (three chorioretinitis and four cortical blindness), but not all had full assessment.

The appearance of the chorioretinitis usually differs from that in toxoplasmosis as in CMV the retina is primarily involved and it is more truly a retinitis which usually becomes inactive in early infancy. An acute retinitis appears as scattered white dots which coalesce and become haemorrhagic and there is often a retinal vas- culitis with arterial sheathing and perivascular exudates. The pigmentation of scars is less than in toxoplasmosis ${ }^{10}$ (see Fig. 1). CMV chorioiretinitis has not been reported to reactivate.

Other less common findings are optic atrophy, ${ }^{11}$ optic disc malformations, ${ }^{12}$ microphthalmos, keratitis, cataracts and anterior chamber malformations with glaucoma. ${ }^{13}$ As CMV is such a common intrauterine infection some of these findings may be just chance associations.

\section{VARICELLA/CHICKENPOX}

Chickenpox (varicella) is common in childhood only about $10 \%$ of women of childbearing age are seronegative. ${ }^{14}$ Chickenpox is the manifestation of primary infection with the varicella-zoster virus $(\mathrm{V}-\mathrm{Z})$. Zoster is caused by the same virus. Like other herpes viruses $\mathrm{V}-\mathrm{Z}$ may persist in a latent form. Zoster is the reactivation of virus previously dormant in a dorsal root ganglion nerve cell. Zoster is only thought to be accompanied by a viremia and generalised rash in patients with reduced cell-mediated immunity.

The $\mathrm{V}-\mathrm{Z}$ virus has been shown to induce chromosomal abnormalities in vitro and in vivo. Chromosomal defects have not been reported in babies born to infected mothers, but there is some evidence that there may be increased risk of childhood leukaemia. The V-Z virus may be transmitted across the placenta, although most infants of women with gestational varicella are born healthy. ${ }^{15}$ There is preference for skin, lung and liver involvement. A pattern of skin scarring, limb hypoplasia brain and eye damage is related to intrauterine $\mathrm{V}-\mathrm{Z}$ infection. This has been called 'The congenital varicella syndrome', first described by Laforet and Lynch. ${ }^{16}$ This syndrome is seen both in infants born to mothers with chickenpox and zoster during pregnancy. However, it is more rare in gestational zoster

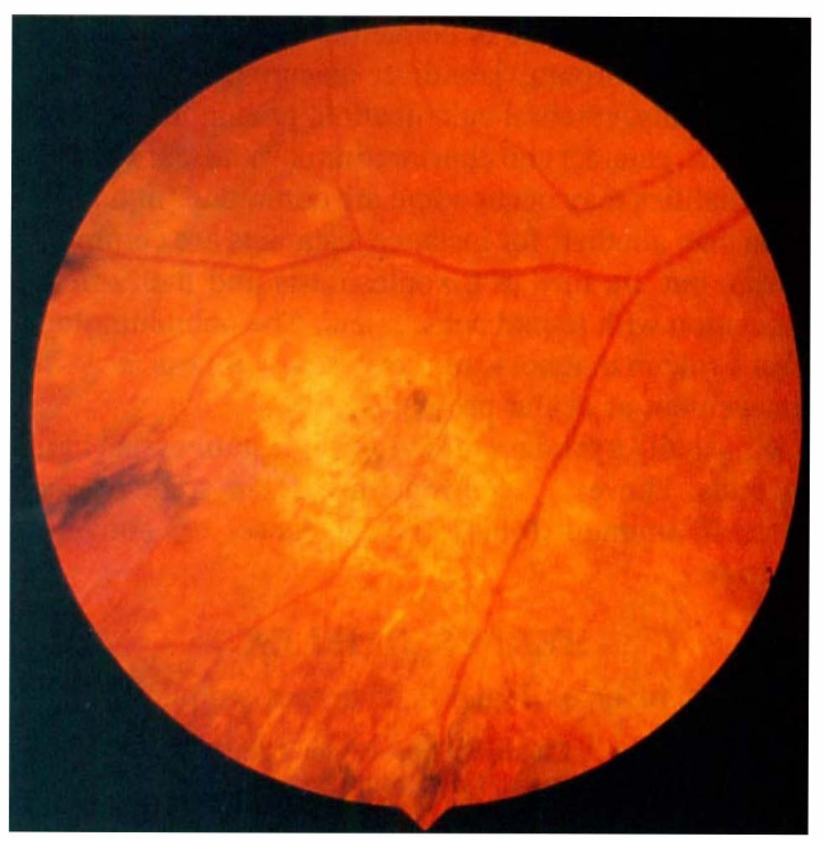

Fig. 1 Fundus photograph showing retinal atrophy and scarring after infection with cytomegalovirus. 
presumably as there is often no associated viremia. Zoster may be commoner during pregnancy, when cell-mediated immunity is reduced as compared with other similarly aged adults.

Virus has not been isolated from the cerebrospinal fluid or from the eye after birth, presumably because virus replication occurred long before this and no active virus is still present.

The incidence of the congenital varicella syndrome is low in infants born to infected mothers. Detection of fetal involvement is often difficult and therefore pregnancy is not usually electively terminated. Limb hypoplasia may be diagnosed in utero by the use of ultrasound. Passive immunisation can conferred by administration of $\mathrm{VZ}$ immunoglobulin from normal donors with high antibody titres to $\mathrm{V}-\mathrm{Z}$ virus. Pregnant women may suffer more severely from virus infections, perhaps due to reduced cell-mediated immunity and chickenpox may be complicated by pneumonitis and death. There are reports of apparently successful drug treatment of infected pregnant women using Acyclovir (ACV). ${ }^{17}$

Gershon summarised the findings in 37 infants with symptomatic congenital infection with the $\mathrm{V}-\mathrm{Z}$ virus and noted ocular abnormalities in $62 \%$ of cases. ${ }^{18}$ The most frequently reported ocular abnormality is chorioretinitis. Typically the lesions are heavily pigmented at their edges with a depigmented centre, very similar to those of congenital toxoplasmosis. There appears to be a predilection for macular involvement, but these lesions are more likely to be detected as they cause symptomatic ocular morbidity and are easily visualised. The retina may be congenitally detached. The eye with congenital varicella cataract is often small with signs of intraocular inflammation such as posterior synechiae. The lens may become calcified. Other ocular features are: nystagmus, microphthalmos, optic atrophy and optic nerve hypoplasia in association with an ipsilateral macular chorioretinal scar. ${ }^{13}$

Neurological lesions in the congenital varicella syndrome include cortical atrophy ( \pm focal calcification), porencephaly, cerebellar hypoplasia, encephalitis, seizures, hemiparesis, hemianopic visual field defect, atrophy of anterior columns of spinal cord in region related to skin scarring, Horner's syndrome, oculomotor palsy, bulbar palsy and neuropathic bladder.

\section{RUBELLA}

Rubella is a member of the togavirus family. There appears to be only one antigenic type and apparent differences in virulence of epidemics is probably a reflection of different population susceptibility.

In 1941 Gregg, an Australian ophthalmologist, first associated intrauterine rubella infection with congenital cataract and heart disease. ${ }^{19}$ The availability of vaccines which seem to confer long-lasting immunity has reduced the incidence of rubella in the developed world, but not erradicated congenital rubella infection. However, recent reports suggest that there has been an increase in incidence in the United States. ${ }^{20}$ The rubella syndrome remains the leading preventable cause of childhood blindness in many regions of the world. ${ }^{21}$

Rubella is transmitted to the fetus during primary infection of the mother. Transmission of virus in an immunised mother or with prior infection with rubella is extremely rare. ${ }^{22}$ Congenitally infected infants shed virus for several weeks after birth and are a reservoir of infection for others. The fact that live virus is still present particularly in brain tissue raise the possibility of chronic cell damage.

There is a very high risk of major congenital defect, spontaneous resorption or abortion in fetal infections during the first eight weeks of pregnancy. If infection occurs before 12 weeks of gestation it more likely to result in cataract as the maximum blood supply to the lens is between two to 11 weeks gestation.

Congenital rubella is often subclinical in neonates, defects, such as hearing loss and mental impairment, may not be manifest until later in life, particularly in infants infected after the 12th week of gestation. Fetuses exposed after 20 weeks of gestation seem not to suffer from congenital defects. Prenatal diagnosis can be made by detection of rubella specific IgM antibodies in fetal blood. ${ }^{23}$

The mechanism of organ damage in the fetus is not known. The predominant histological feature is a vasculitis. Recent reports suggest that rubella interferes with mitosis, ${ }^{24}$ reducing cell multiplication when turnover is usually very high during organogenesis.

The most frequent clinical findings in the congenital rubella syndrome are: intrauterine growth retardation, hepatosplenomegaly, pulmonary arterial hypoplasia, patent ductus arteriosus, interstitial pneumonitis, thrombocytopenia, encephalitis, hearing defects, retinopathy and cataracts.

The most common eye finding is a pigmentary retinopathy which is usually bilateral. ${ }^{25,26}$ The mottled pigmentation is most marked at the posterior pole. The retinal vessels are normal in calibre. If the retinopathy is not complicated by cataract the electroretinogram rarely is abnormal ${ }^{27}$ and the electrooculogram is unaffected. ${ }^{28}$ The retinopathy of rubella is usually consistent with near normal acuity, ${ }^{29}$ but subretinal neovascularisation may later impair central acuity. Hypertensive retinopathy may develop in association with renal artery and aortic stenosis. ${ }^{20}$

Cataracts are often bilateral ( $81 \%$ in Wolff's series $\left.{ }^{26}\right)$. Commonly there is a central pearly white opacity with a clear peripheral zone. The cataracts may become progresively denser after birth or spontaneously resorb. ${ }^{31}$ Histology of the lens typically shows persistence of the nuclei in the central fibres, suggestive of retarded development. ${ }^{32}$ The eye is frequently moderately microphthalmic. There is a higher incidence of postoperative uveitis, posterior synechiae and pupil block glaucoma than in surgery for congenital cataracts of other aetiologies. Virus can be isolated from lens aspirates. Hypoplasia of the stroma and the dilator muscle of the iris is common, making lens surgery more difficult. Corneal haze is usually transient, but more rarely associated with congenital glaucoma which is 
usually bilateral. ${ }^{33}$ Arrested development of the anterior chamber angle has been reported.

Infantile or juvenile glaucoma may develop in patients without cataract or buphthalmos and there is an increased incidence of keratoconus with corneal hydrops. There is a high incidence of refractive error, both hypermetropia and myopia.

\section{TOXOPLASMOSIS}

Toxoplasma gondii is a common infection of man and other animals. It is a coccidian and exists in three forms. The oocyst form is excreted in cat faeces. These may contaminate unwashed human food. Human infection may also occur with the tissue cyst form, particularly in incompletely cooked meats. The tacchyzoite form of the parasite is seen in the acute infection.

In the adult infection with toxoplasma gondii is often asymptomatic. In this country $20 \%$ of pregnant women are seropositive at their first antenatal visit as compared with $80 \%$ in France. ${ }^{34}$ There is recent evidence that recurrent as well as primary infection of the mother may result in infection of the fetus. ${ }^{35}$ Spiramycin has been advocated for treatment of infected mothers as it is believed to concentrate in the placenta, but it is not available in the UK and its benefit has not been proven.

In the congenitally infected infant the organism may become widely disseminated. The major organ damage that occurs in some congenitally infected infants may be due to specific deficits in cell-mediated responses to Toxoplasma lysate antigens. ${ }^{36}$ Encephalitis and hepatosplenomegaly are the major features. There is often microcephaly and there may be intracranial calcification. Hydrocephalus can coexist with microcephaly and porencephaly leading to difficulty in its diagnosis. Longterm problems in survivors are seizures, developmental delay, microcephaly, deafness and visual loss. The most severe disease seems to occur in those infected during the first trimester.

The commonest manifestation of congenital infection is chorioretinitis. The typical chorioretinal scars have a circumscribed hyperpigmented border with a pale centre which may be raised (see Fig. 2). They seem to have a predilection for the macular region and the infant may only present later when a squint develops or poor acuity is detected. Toxoplasma organisms have been identified in the optic nerve $\mathrm{e}^{37}$ and in the nerve fibre layer of the retina ${ }^{38}$ and may even spread by this route explaining the preponderance of macula lesions. Histologically toxoplasma lesions show an intensely necrotising retinitis with inflammation of the underlying choroid. ${ }^{39}$

Chorioretinitis may be severe presenting at birth with leukocoria, a dense yellow retrolental mass with overlying white dots and blood vessels. The anterior segment shows signs of chronic inflammation (keratitis and posterior synechiae). The eye may be small, with persistent pupillary membrane and hyaloid artery suggesting arrested ocular development as a result of the infection. Ultrasonography may reveal a solid mass in the posterior segment and funnel shaped retinal detachment. ${ }^{40}$
Orbital cellulitis with chorioretinitis has been reported in one case. ${ }^{40}$ Neurologic and ophthalmic lesions may be quiescent with the parasite in an encysted form only to become active several months or years later when seizures, hydrocephalus or visual problems become manifest in a previously apparently healthy individual. Reactivation of the chorioretinal lesion appears as 'fluffy' white area in the old scar with vitritis. Reactivation of lesions is probably partly due to a hypersensitivity phenomenon ${ }^{41}$ as well as multiplication of tachyzoites within the retina. ${ }^{42}$ Neovascular membranes may develop at the edge of old scars causing central visual loss.

\section{SYPHILIS}

The organism of congenital syphilis is Treponema pallidum. Since the discovery of penicillin, syphilis has become much less common in the western world, but there were 365 cases of less than one year of age in the United States in $1986 .^{43}$

Infection spreads from the placenta in the fetal bloodstream. The lesion of syphilis in all tissues is of a perivascular infiltration of lymphocytes, plasma cells and histiocytes, with obliterative endarteritis and extensive fibrosis. The lesions are only seen after the 16th week of gestation when the fetal immune system first produces its response to the infection. The infant is often stillborn with massive organ involvement but milder infections can present as failure to thrive with persistent rhinitis, hepatosplenomegaly and maculopapular rash. The meninges may be involved with progressive hydrocephalus and cranial nerve palsies as a result. Treatment is with two weeks of intravenous penicillin.

The ocular manifestations in the infant include a 'salt and pepper' fundus, uveitis, glaucoma, optic atrophy and rarely chancres of the eyelid. The pigmented dots are

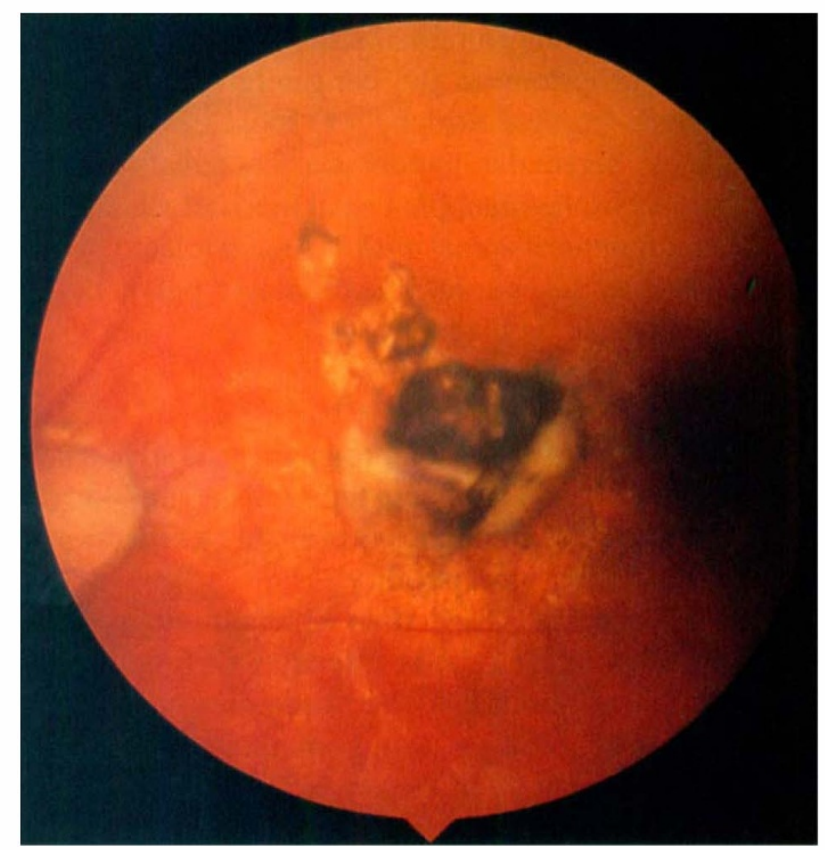

Fig. 2 Fundus photograph to show a scar of inactive toxoplasmosis at the macula. 
intermingled with yellowish red spots. The retinal pigment changes are most marked in the periphery and may mimic retinitis pigmentosa, with attenuation of the vessels and pallor of the optic disc in severe cases. Focal granulomata rarely occur in the anterior chamber.

In untreated patients older than five years of age the classic Hutchinson's triad of interstitial keratitis, nerve deafness and notched incisor teeth is seen. The keratitis presents with acute photophobia, lacrimation, corneal oedema and iritis. It is a hypersensitivity reaction and responds to topical corticosteroids, leaving corneal scarring, ghost vessels and iris atrophy.

\section{CONCLUSIONS}

Whilst we recommend the traditional TORCH screen for IgG to Toxoplasma, Rubella, CMV and Herpes simplex in infants with features of congenital infection, this approach should not discourage careful clinical assessment, particularly ophthalmic examination, to characterise distinguishing features which may lead to further serological tests such as for varicella zoster or syphilis. In particular any infant with a rash suggestive of herpes simplex should have a fundus examination as the consequences can be blinding.

If the infective agent is still not identified the search should continue, parvovirus, enterovirus and listeria to give just a few examples, are becoming associated with congenital infection and their role in eye pathology is not yet known.

Early diagnosis and treatment is particularly important as in many intrauterine infections progressive neurological damage occurs with hearing and visual loss. Hopefully, prevention by immunisation programmes and treatment of infected pregnant women will make these conditions the rarity that congenital syphilis for the time being still is. With the advent of HIV infection unfortunately we expect a rise in the incidence of syphilis and are already seeing an increase in the number of infants born with symptomatic CMV.

Key words: Cytomegalovirus, Eye, Herpes simplex, Intrauterine infection, Review, Rubella, Syphilis, Toxoplasma gondii, Varicella-zoster.

\section{REFERENCES}

1. Editorial: TORCH syndrome and TORCH screening. Lancet 1990, 335: 1559-61.

2. Cibis A and Burde RM: Herpes simplex virus induced congenital cataracts. Arch Ophthalmol 1971, 85: 220-6.

3. Dahle AJ and McCollister FP: Audiological findings in children with neonatal herpes. Ear-Hear 1988, 9(5): 256-8.

4. El Azazi M, Malm G, Forsgren M: Late ophthalmologic manifestations of neonatal herpes simplex. Am J Ophthalmol 1990, 109(1): 1-7.

5. Stagno S: Cytomegalovirus. In: Infectious diseases of the fetus and newborn infant, 3rd edition. Eds. Remington and Klein, WB Saunders, Philadelphia 1990, Chapter 6, pp. 241-81.

6. Gehrz RC, Peterson ES, Liu YN: Immune mechanisms in congenital cytomegalovirus infection: activation of CMVspecific $\mathrm{T}$ helper cells (CMV-Th) by exogenous IL-2. Clin Exp Immunol 1988, 74: 333-8.
7. Belec L, Tayot J, Tron P, Mikol J, Scaravilli F, Gray F: Cytomegalovirus encephalopathy in an infant with congenital acquired immuno-deficiency syndrome. Neuropediatrics 1990, 21: 124-9.

8. Stagno S, Pass RF, Dworsky ME et al.: Congenital and perinatal cytomegaloviral infections. Semin Perinatol 1983, 7: $31-42$.

9. Ramsay MEB, Miller E, Peckham CS: Outcome of confirmed symptomatic congenital cytomegalovirus infection. Arch Dis Child 1991, 66: 1068-9.

10. Boniuk I: The cytomegalovirus and the eye. Internat Ophthalmol Clin 1972, 12: 169-90.

11. Tarkkanen A, Merenmies L, Holmström T: Ocular involvement in congenital cytomegalic inclusion disease. J Pediatr Ophthalmol 1972, 9: 82-6.

12. Hittner HM, Desmond MM, Montgomery JR: Optic nerve manifestations of human congenital cytomegalovirus infection. Am J Ophthalmol 1976, 81: 661-5.

13. Lambert SR, Taylor D, Kriss A, Holzel H, Heard S: Ocular manifestations of the congenital varicella syndrome. Arch Ophthalmol 1989, 107: 52-6.

14. Gershon AA, Raker R, Steinberg S, Topf-Olstein B, Drusin LM: Antibody to varicella-zoster virus in parturent women and their offspring during the first year of life. Pediatrics 1976, 58: 692-6.

15. Siegel M: Congenital malformations following chickenpox, measles, mumps and hepatitis. Results of a cohort study. JAMA 1973, 226: 1521-4.

16. Laforet EG and Lynch CL Jr: Multiple congenital defects following maternal varicella: Report of a case. N Eng J Med 1947, 235: 534-7.

17. Landsberger EJ, Hager WD, Grossman JH: Successful management of varicella pneumonia complicating pregnancy. A report of three cases. J Reprod Med 1986, 31: 311-14.

18. Gershon AA: Chickenpox, measles and mumps. In: Infectious diseases of the fetus and newborn infant, 3rd edition. Eds. Remington and Klein, WB Saunders, Philadelphia 1990, Chapter 11, pp. 395-445.

19. Gregg NMCA: Congenital cataract following German measles in the mother. Trans Ophthalmol Soc Aust 1941, 3: $35-44$.

20. Increase in rubella and congenital rubella syndromeUnited States, 1988-1990. MMWR 1991, 40(6): 93-9.

21. Moriarty BJ: Childhood blindness in Jamaica. $\mathrm{Br} \mathrm{J}$ Ophthalmol 1988, 72: 65-7.

22. Morgan-Capner P: Does rubella re-infection really matter? In: Mortimer PP, ed. Public health virology: 12 reports. London: Public health laboratory service, 1986: 50-62.

23. Freij BJ, South MA, Sever JL: Maternal rubella and the congenital rubella syndrome. Clin Perinatol 1988, 15: 247-57.

24. Bowden DS, Pedersen JS, Toh BH et al.: Distribution by immunoflourescence of viral products and actin-containing cytoskeleton filaments in rubella virus-infected cells. Arch Virol 1987, 92: 211-19.

25. Marks EO: Pigmentary abnormality in children congenitally deaf following maternal German measles. Trans Ophthalmol Soc Aust 1946, 6: 122-5.

26. Wolff SMcK: The ocular manifestations of congenital rubella. Trans Am Ophthalmol Soc 1972, 70: 577-614.

27. Obenour LC: The electroretinogram in rubella retinopathy. Internat Ophthalmol Clin 1972, 12: 105-10.

28. Krill AE: The retinal disease of rubella. Arch Ophthalmol 1967, 77: 445-49.

29. Collis WJ and Cohen DN: Rubella retinopathy. A progressive disorder. Arch Ophthalmol 1977, 84: 33-5.

30. Fortuin MJ, Morrow AG, Roberts WC: Late vascular manifestations of the rubella syndrome. Am J Med 1971, 51: 134-40.

31. Smith GT, Shun-Shin GA, Bron AJ: Spontaneous reabsorp- 
tion of a rubella cataract. $\mathrm{Br} J$ Ophthalmol 1990, 74: 654-65.

32. Zimmerman LE: Pathogenesis of rubella cataract: Gregg's syndrome. Arch Ophthalmol 1965, 73: 761-3.

33. Sears ML: Congenital glaucoma in neonatal rubella. $\mathrm{Br} J$ Ophthalmol 1967, 51: 744-8.

34. Ruoss CF and Bourne GL: Toxoplasmosis in pregnancy. $J$ Obstet Gynaecol 1972, 79: 1115-18.

35. Fortier B, Aïssi E, Ajana F, Dieusart P, Denis P, Martin de Lassalle E, Lecomte-Houcke M, Vinatier: Spontaneous abortion and reinfection by toxoplasma gondii (letter). Lancet 1991, 338: 444.

36. McLeod R, Mack DG, Boyer K, Mets M, Roizen N, Swisher C, Patel D, Beckman E, Vitullo D, Johnson D et al:: Phenotypes and functions of lymphocytes in congenital toxoplasmosis. J Lab Clin Med 1990, 116: 623-35.
37. Fair JF: Clinical eye findings in congenital toxoplasmosis. Surv Ophthalmol 1961, Supple 832-838.

38. Zimmerman LE: Ocular pathology of congenital toxoplasmosis. Surv Ophthalmol 1961, Suppl 832-838.

39. Tabbara KF: Ocular toxoplasmosis. Int Ophthalmol 1990, 14(5-6): 349-51.

40. Pettapiece MC, Hiles DA, Johnson BL: Massive congenital toxoplasmosis. J Pediatr Ophthalmol 1976, 13: 259-65.

41. Frenkel JK: Pathogenesis of toxoplasmosis and of infection with organisms resembling Toxoplasma. Ann N Y Acad Sci 1956, 64: 215-51.

42. O'Connor GR: Ocular toxoplasmosis. Trans New Orleans Acad Ophthalmol 1983, ??: 108-212.

43. Mascola L, Pelosi R, Blount JH, Alexander CE, Cates W: Congenital syphilis revisited. Am J Dis Child 1985, 139; 575-80. 